\title{
THE CONCEPT OF INERTIA: AN INTERDISCIPLINARY APPROACH
}

Paolo Bussotti

University of Udine, Italy

\begin{abstract}
"Every body perseveres in its state of rest, or of uniform motion in a right line, unless it is compelled to change that state by forces impressed thereon" (p. Newton 1846, p. 83).

This is the famous first axiom or law of motion stated by Newton in his masterpiece The Mathematical principles of natural philosophy (ivi). Everywhere, in the courses of physics at the high school level the inertia principle is the first to be taught. However, there are many doubts that most of learners fully grasp its numerous and fundamental nuances, which are necessary for a satisfying introduction to physics. Therefore, I propose an interdisciplinary approach for the explanation of this principle in which history of science and analysis of the daily experiences are joined to offer a complete comprehension of the concept of inertia.
\end{abstract}

Most of the young people aged 15-16 years are tied to their immediate sensorial experiences, which do not induce to think that the inertia law might be valid: if you throw an object, you observe that it does not prosecute along a straight line, but it tends to fall on the ground following a strange trajectory. At all appearances, the trajectories of the objects are different according to the material of which the object is composed. Also, in conditions in which apparently no impressed force exists, the movements of the objects are anything but uniform and rectilinear. If a learner is interested in the movements of the skies -also granted that he/she knows the Copernican system -, he/she will be impressed by the regularities of such movements, which, certainly, are not rectilinear uniform. But which force can exist in the skies that makes the bodies to deviate from a rectilinear path travelled with uniform speed? Let us suppose that a young be extremely gifted, that he/she is able to overcome all these difficulties: there will be, however, a crucial difficulty which very likely will be not solved in the learner's mind. It is so expressible: "dear teacher, you speak of rest and uniform motion in a right line, but in respect to what do you determine these kinetic states? For me, in my car, I am at rest and the external world is moving; for the external world, I am moving. What is the situation?"

No fear, no hurry: the same questions and doubts which are in the minds of the pupils were also in the minds of the scientists of the $16^{\text {th }}$ and $17^{\text {th }}$ centuries, and they solved them in the admirable period between 1543 (Copernicus published The revolutions of the celestial spheres, Copernicus 1978) and 1687 (Newton published The Mathematical principles of natural philosophy, Newton 1846). This is the golden age of Scientific Revolution.

To recall in synthesis the steps through which the scientists overcame such difficulties is the best way to approach the basic concepts of physics. Furthermore, this will allow the teachers to connect the lessons of physics with those of history and philosophy (in the countries where this discipline is taught at high school level). This is a profound interdisciplinary approach.

Let us divide this didactical proposal into steps:

1. Relativity of the motions. It is necessary to clearly explain that no body is at rest or in motion in an absolute sense. It depends on the choice you do. In most cases, for us it is convenient to consider the Earth at rest, though we know that it is not a rest. Our sensorial experience is misleading because it induces us to think the Earth to be at rest. However, if the teacher is careful, some sensorial and visual experiences can indicate the relativity of motion. Each of us has experienced that when a train starts slowly, the passengers have the impression that the train remains at rest and that the station is moving. History of science can be used to avoid that the concept of 
relativity of motion be trivialised. It is appropriate to remember that Aristotle in his Physics thought the Earth to be absolutely at rest, and that the kinetic states of the other bodies should (not might) be determined in respect to the Earth. Though not all of the scientists shared Aristotle's view, his Physics was one of the most successful books in the scientific-philosophical panorama of the Antiquity and Middle Ages. Copernicus felt the need to highlight the relativity of the motions (he was not the first one to guess such relativity, but in his epoch, it was not granted at all). Copernicus wrote:

Every observed change of place is caused by a motion of either the observed object or the observer or, of course, by an unequal displacement of each. For when things move with equal speed in the same direction, the motion is not perceived, as between the observed object and the observer, I mean. It is the earth, however, from which the celestial ballet is beheld in its repeated performances before our eyes. Therefore, if any motion is ascribed to the earth, in all things outside it the same motion will appear, but in the opposite direction, as though they were moving past it (Copernicus 1978, book l, chapter 5, pp. 11-12).

Thus, a recall to history is a good means for the pupils to understand that the concepts of physics are at the basis of our view of the world and that without the notion of relativity of the movements, the Copernican system would have been impossible. This is of help to guess the interconnections among knowledge which, at a superficial view, might appear unrelated but that, in fact, are. Of course, collaboration with the teacher of History might be conceived to insert Copernicus' thought within the picture of the Renaissance.

2. Approach to inertia. After having spoken of the relativity of any motion, the teacher of physics might enter the classroom developing this experiment without any initial explanation. Let us consider two joined equal inclined planes as in the Figure 1:

\section{Figure 1}

First Phase of the Experiment: Two Inclined Planes with the Same Inclination

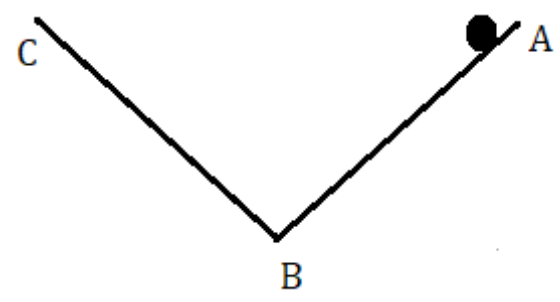

A little ball is in A at the altitude $h$. If you precise that the two planes have to be considered completely smooth, probably all of the learners will intuitively claim that when the ball has reached the point $B$, it will ascend on the plane $B C$ in $C$ at the same altitude it had in $A$, not higher, not lower.

Let us not suppose that the plane $B C^{\prime}$ is less inclined than $A B$ on the horizon, as in the following Figure 2.

\section{Figure 2}

Third Phase of the Experiment: Two Inclined Planes with Different Inclinations




The learners will recognise that the little ball will arrive at $C^{\prime}$ at the same altitude it had in $A$, not higher, not lower.

Finally, suppose that the plane $\mathrm{BC}^{\prime}$ becomes always less inclined on the horizon until becoming perfectly horizontal like this (Figure 3).

\section{Figure 3}

Third Phase of the Experiment: Inclined Planes of Various Inclinations until a Horizontal Plane

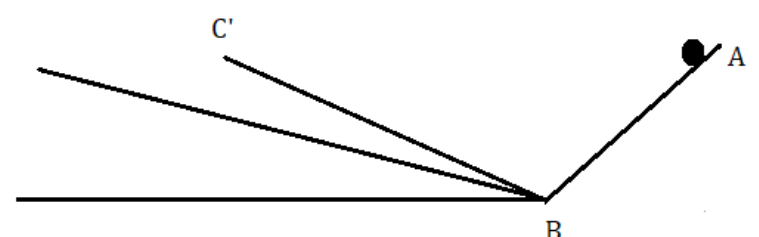

Now ask the learners: consider the situation in which the second plane is completely horizontal; the ball arrives at $B$, what happens then? Probably most of them will answer correctly: the ball arrives at B with a certain speed, after that it continues its motion along the horizontal plane maintaining the same speed as in B. There is no sufficient reason because something different happens. Well, you will tell your pupils: "very good, you have arrived on your own at the inertia principle. Bravo! Not only: you have followed the same train of thoughts through which Galileo reached the concept of rectilinear inertia". For, Galileo in the Second Day of his Dialogue Concerning the Two Chief World Systems (1632, Galilei 1967) developed a reasoning whose synthesis has been just described. The teacher might read this important passage of the Dialogue (ivi, pp. 145-146) to the class, so that the learners, through Galileo's detailed descriptions and explanations, could realise all the nuances of this concept and the difficulties encountered by the most brilliant minds of mankind to reach it.

Thus, the human aspect of science will be also pointed out: the scientists have reached the notions which nowadays guide our knowledge of the world through a difficult road, full of obstacles. Only their hard work permitted to overcome the obstacles. A clever mind is nothing without the work.

The way in which Galileo (and the students!) have reached the idea of inertia allows us to develop some interesting conceptual and didactical considerations:

a) The whole reasoning relies on the assumption that the surfaces of the two inclined planes are perfectly smooth, without any friction. In fact, no such surface exists in the reality. No space devoid of any friction exists. What does this mean? Is the inertia principle not valid? Here, the teacher has to be careful to distinguish the world and our model of the world. The inertia principle belongs to our model of the world. It shows a tendency of the real bodies, which is indispensable to understand their kinetic behaviour. It is clear that in reality no motion is perfectly inertial. The model has to offer a scheme in which, abstracting from some properties $P$ of the bodies, we are able to focus our attention on properties $Q$ we consider more profound. In the reality $P$ and $Q$ cohabit. In the case of inertia, the property $P$ is represented by the existence of friction, constrains and obstacles, the property $Q$ is the inertial tendency of the rectilinear uniform motions. If we wanted to study the motions under the condition that a friction exists, we will include the friction in the properties $Q$ and so on. Each situation analysed by physics deals with our theoretical model which is a simplified view of reality. Of course, our model is the more satisfactory the more aspects of reality it will explain and foresee, but every model will abstract from some aspects of reality.

b) Our experiment did not demonstrate the mathematical, logical validity of the inertia principle. No such demonstrations can exist in classical physics, because, as the name itself clarifies, that of inertia is a principle, an axiom, a postulate; it is not proved through a series of reasonings. Rather, it is one of the axiomatic bases to develop theorems and propositions. Galileo's and our experiments make the principle plausible, reasonable; but do not demonstrate it. Newton was perfectly right to assume inertia principle as an axiom.

c) Since a uniform rectilinear motion continues without the need of any external action, this means that such an action - which the physicists from Newton onwards call force - has not to produce velocities, 
but change of velocities, that is accelerations (I give for granted that the learners know the vectors' elementary properties). Let us now see, the basis of the concept of force.

3. Forces. In the world you observe that most motions are not uniform and rectilinear. Therefore, inertia principle is not sufficient in our physics. It is necessary to identify an action which modifies the kinetic state of the bodies. It is clear that such action has to be proportional to acceleration. Newton identified the proportionality factor which ties force and acceleration with the mass (considered as quantity of matter) of a body (Definition VIII of Newton's Principles). From here the famous formula $\boldsymbol{F}=m \boldsymbol{a}$. This definition of force seems reasonable: after all, from an intuitive standpoint, if we have two objects - let us suppose two carts - of the same form and of the same material, we struggle the more to move the cart the bigger it is, because it has more mass, more matter than the smaller one. This definition of force seems acceptable also considering the forces which produce circular motions. Consider a sling and a stone at its extremity. We make to rotate the sling. As in the previous case, we struggle to move the sling the bigger the stone is. At all appearances, things work perfectly. Following Newton, you will identify the inertial mass (he called it innate force, vis insita, Definition III) with the resistance of a body to modify its state of rest or of uniform rectilinear motion. This is fully coherent with the general definition of mass as quantity of matter: the more massive a body is, the more it is difficult to modify its inertial state. Now, following this partially historical itinerary, you are ready to introduce the third Newton's principle of dynamics: to any action an equal and opposite reaction corresponds. This is intuitive as well: if you hit a wall, you feel a pain, it is as if the wall hit you.

The learners should have no difficulty to understand the order of thoughts traced until now. Some elements of the history of physics have been useful to highlight the kind of difficulties the learners can encounter as well as their solutions.

However, something is missing in our construction and something very important: the forces we have introduced are supposed to act through a direct contact, but on the Earth, there is another action, which acts on all the bodies independently of their composition, of their form, of their nature. This force is gravity. But gravity does not act through a direct contact. Nonetheless, there is no doubt that it changes the state of motion of a body. Furthermore: what is the usual way in which we measure the mass of a body? Certainly, we do not check how difficult to move a cart is, or how strong the resistance of a stone is when we put a sling in rotation. There is by far a more usual and easier solution: we pose a body on a balance, we read on the balance its weight, and we divide the weight by the acceleration of gravity $\boldsymbol{g}$. Thus, we obtain the mass. This is our normal procedure. It is fully consistent with our definition $\boldsymbol{F}=m \boldsymbol{a}$. In this case $\boldsymbol{a}=\boldsymbol{g}$. But here there is a conceptual problem on which the learners have to reflect from the beginning of their education in physics: if we try to determine the mass of an object through the resistance it opposes to any change of its kinetic state, it seems that we determine something completely different in respect to the mass of the same object considered as the way in which it is attracted by the Earth. This is the gravitational mass of a body, not its inertial mass. Why do we speak of "mass" in both cases? The answer is that classical physics has no answer to this question. If, empirically, you measure the inertial mass and the gravitational mass of the same body, you find the same value for the two masses. Numerous experiments have always given the same results. But in Newton's physics there is no theoretical explanation on the coincidence of the two masses.

The set of these considerations is necessary for the learners to achieve a correct idea of physics, of its conceptual problems and of its theoretical value. They will guess that we cannot claim that science explains everything without posing some unexplained presuppositions which have the role of the axioms in mathematics. Not only: sometimes we have to accept situations as that connected to the problem as inertial mass / gravitational mass which belong neither to the presuppositions nor to the consequences of a theory. It happens accidentally in classical physics, in spite of the fact that the coincidence between the two masses implies fundamental consequences. The learners - or at least some of them - will be curious to know whether a theory which explains such coincidence exists, and whether - more in general - a theory can offer complete explanations of all the phenomena it deals with. You will immediately explain that, though science cannot explain everything, it is the most reliable form of knowledge available for mankind. On these bases, you might also develop a brief reasoning on the general nature of knowledge, which might be very useful and stimulating for the pupils. Thus, the proposed conceptual-historical approach might induce many interesting questions in the minds of the learners.

The inertial reference frames. We have seen that in our common experience every motion is relative, we have seen what inertia is, and we have seen what a force is. Now there is the fundamental and most difficult question: if every 
motion is relative, how is it possible to recognize an inertial kinetic state from one which is not? In other terms: to what should inertia be referred since inertia is such a fundamental property which, being connected to the mass, seems an absolute and not a relative property? This is one of the most difficult conceptual theoretical questions in classical physics. Reflecting on this problem, the learners - before dealing with more specific concepts, problems and developments - will fully grasp the conceptual frame in which classical physics is inserted. Here the historical development plays a decisive role. Newton, at the dawn of modern physics, conceived one of the most genial reasonings ever developed in science (final section of the Definitions in his Principles). He thought to have identified an absolute motion and an absolute space to be considered as the real inertial reference frame. The concept of inertia should be referred to such absolute space. Newton's argument is based on the celebrated bucket experiment. I recommend the teachers to explain this experiment in any detail to their pupils. I will restrict to refer to the experiment and to Newton's interpretations. Broader considerations on this experiment might be the occasion for a further Editorial in our JBSE!

\section{Figure 4}

The Various Phases of the Bucket Experiment

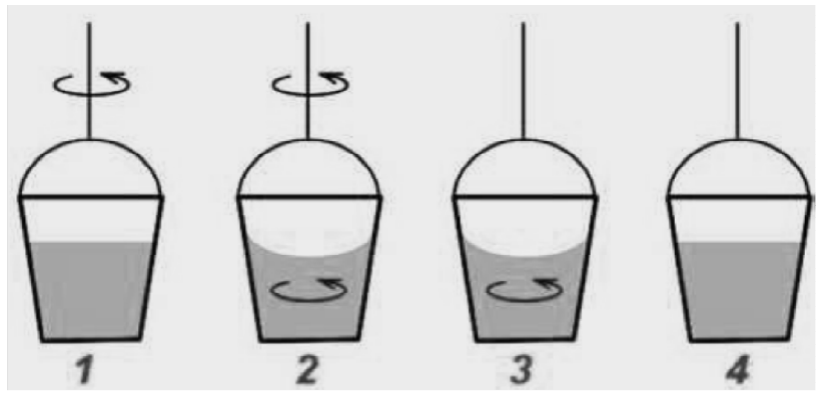

Let us consider a bucket hanging at a rope as in the Figure 4. Act like this:

(1) Twist the rope until it is very hard and keep the rope twisted;

(2) Fill the bucket of water;

(3) Free the rope. You observe that the bucket begins to rotate, but you will observe no movement in the water (picture 1, Figure 4);

(4) After a little bit time the bucket, because of the friction between the particles of the water and those of the bucket's walls communicates the movement to the water, which begins to rotate and to ascend along the bucket's walls (picture 2, Figure 4);

(5) After a while, the bucket stops its movement, but the water continues to rotate (picture 3, Figure 4);

(6) Finally, both the bucket and the water are at rest (picture 4, Figure 4).

Newton interpreted the rotational movement of the water as due to its inertia: during their movement, each particle of water tends to escape along its instantaneous tangent because of the inertia principle. This inertia tendency cannot be actualised because of the bucket's walls. Thence, the particles of water heap up on the bucket's walls.

Once clarified that the ascent of the water's particles is due to their inertia, Newton wondered to what the inertia might be referred. His reasoning can be summarised in the following Table 1:

Table 1

A Summary of Newton's Reasoning

\begin{tabular}{cccc}
\hline & Relative motion water/bucket & Water's surface & Inertial forces \\
\hline 1. & Max & Flat & No \\
2. & No & Curved & Yes \\
3. & Max & Curved & Yes \\
4. & No & Flat & No \\
\hline
\end{tabular}


Is it possible that the inertia of water depends on the relative motion between water and bucket? No, it is not, because you note the effects of the inertial tendency (to summarise, but with a little linguistic impropriety, called "inertial forces") both in the case in which the relative motion water/bucket does not exist (phase 2) and in that in which it is maximal (phase 3). You note the lack of inertial forces both in the case in which the relative motion water/bucket is maximal (phase 1) and in that in which no relative motion exists (phase 4).

Thence, Newton concluded that inertia cannot be referred either to the bucket or, even the less, to other objects close to the water. He referred inertia to an Absolute Space. In this way, the concept of inertia is well founded; it is possible, at least in principle, if not in practice, to distinguish the inertial reference frames from the others: they are the frames which are at rest or move rectilinearly and uniformly in respect to the Absolute Space.

Is this picture completely satisfactory? The answer to a next Editorial!

\section{References}

Copernicus, N. (1978). On the Revolutions. Edited by F. Dobrzycki. Translation and Commentary by E. Rosen. Polish Scientific Publishers and Macmillan Press. Latin edition 1543.

Galilei, G. (1967). Dialogue concerning the two chief world systems. Translation by S. Drake, Introduction by A. Einstein. University of California Press. Second Edition. Italian edition 1632.

Newton, I. (1846). The mathematical principles of natural philosophy. Reprint of A. Motte's Translation (1727). Daniel Adee. Three Latin editions: 1687, 1713, 1726. 